\title{
PRESENCIA DE LOS BLOGS EN EL ÁMBITO DE LA BIBLIOTECONOMÍA Y DOCUMENTACIÓN EN ESPAÑA: ¿SE PUEDE HABLAR DE UNA "BIBLOGSFERA"?
}

\author{
Miguel Ángel Vera Baceta* \\ FOREM Murcia
}

\begin{abstract}
Resumen: El fenómeno blog ha provocado una forma natural de agrupación y comunicación entre aquellos que tienen inquietudes afines. Dentro del ámbito científico ha sucedido de la misma manera, creando comunidades de especial interés en un contexto en el que los blogs se consolidan como fuente de información y herramienta complementaria a la investigación. Si bien la libertad por parte del autor a la hora de ofrecer y organizar sus contenidos puede considerarse una de las principales virtudes y factor desencadenante del éxito de los blogs, también se convierte en uno de sus principales problemas. Los blogs, sus contenidos y sus comunidades son difícilmente identificables, clasificables y cuantificables, constituyendo un todo difuso bajo la denominación de blogosfera que en algunos casos impide la puesta en valor de su contenido. Mediante este artículo se pretende identificar los blogs relacionados con la Biblioteconomía y Documentación en España, su autoría, sus contenidos y sus relaciones para clarificar si se puede realmente hablar de una Biblogsfera.
\end{abstract}

Palabras clave: Bitácoras; blogs; blogosfera; biblogsfera; acceso abierto; España, Biblioteconomía y Documentación.

Tittle: THE PRESENCE OF BLOGS IN THE FIELD OF LIBRARY AND INFORMATION SCIENCES IN SPAIN: CAN WE TALK ABOUT THE EXISTENCE OF A "BIBLOGSFERA"?

Abstract: The blog phenomenon has meant a natural way of grouping and establishing communication amongst those who have similar concerns. This has happened the same way in the field of science, thus creating communities of special interest in a context in which blogs are consolidating as sources of information and extra tools for research. While the authors' freedom to organise and offer their contents may be considered one of the main advantages and a trigger for the success of blogs, it is also one of their main drawbacks. The contents and communities of blogs are not easy to identify, classify and quantify, so that they constitute a vague concept under the name of "blogosphere". This article is meant to identify the blogs related to the field of Library and Information Sciences in Spain, their authorship, their contents and their connections in order to clarify if we can really talk about the existence of a "Biblogsfera".

Keywords: Blogs; blogosphere; biblogsfera; open access; Spain; Library and Information Science.

\section{INTRODUCCIÓN.}

Los blogs están ganando la batalla en lo que a publicación de contenidos en la web se refiere. De la mano de plataformas como WordPress, bajo la etiqueta de CMS (Content Management System), características como su simplicidad, su sencillo manejo y su facilidad a la hora de publicar han hecho que se extiendan de manera generalizada, de hecho, WordPress cuenta ya con más de 74 millones de blogs en todo el mundo ${ }^{1}$.

En cualquier caso, los blogs van más allá de la estricta publicación de contenidos en la web. Estos se enmarcan en el ámbito de las redes sociales gracias a su patrón de comunicación inmediato, interactivo, multidireccional y descentralizado, modelo que ha provocado, en muchas ocasiones, la agrupación, de manera espontánea, de autores y usuarios con algún tipo de afinidad o interés común.

Todas estas características hacen de los blogs una herramienta con grandes cualidades para el ámbito científico, hecho que no ha pasado inadvertido, siendo asimilados de forma natural por algunos de sus autores que, de la misma manera, se agrupan en comunidades y que utilizan estos blogs para mantenerse interconectados, actualizados y como herramienta de discusión o como termómetro de interés y tendencias.

Pero, ¿qué le da carácter científico a un blog? Según Torres y Cabezas (2008) esta respuesta se puede plantear desde al menos dos perspectivas: la primera, desde el punto de vista del contenido, esto es, que el blog hable sobre una temática científica; y la segunda, siguiendo a otros autores como Zivkovic (2006), el carácter científico lo daría la autoría, debiendo estar formada por algún miembro de la comunidad científica y académica con cierto grado de competencia.

*verabaceta@gmail.com

Recibido: 27-01-2014; $2^{\mathrm{a}}$ versión: 25-02-2014; aceptado: 26-02-2014.

VERA BACETA, M.A. Presencia de los blogs en el ámbito de la Biblioteconomía y Documentación en España: ¿se puede hablar de una Biblogsfera? Anales de Documentación, 2014, vol. 17, $\mathrm{n}^{\circ}$ 1. Disponible en: http://dx.doi.org/10.6018/analesdoc.17.1.191141 
Así, Torres y Cabezas hacen referencia a otras funciones de los blogs científicos como medio de publicación sin intermediarios, como tablón de anuncios y repositorio personal o colectivo, como medio de difusión selectiva de la información y para acercar la ciencia al público no especializado (Torres y Salinas, 2008).

Desde esta perspectiva, identificado un blog científico y haciendo una analogía de su funcionamiento y contenido con la tendencia actual hacia el Acceso Abierto de la información científica ${ }^{2}$, Swan (2013) resalta como principales aspectos de dicho Acceso Abierto (SWAN, 2013):

- Que mejora la velocidad, eficiencia y eficacia de la investigación

- Que permite la investigación interdisciplinar

- Que permite hacer cómputos a partir de la literatura científica

- Que aumenta la visibilidad, uso e impacto de la investigación

- Que permite a las comunidades de profesionales, expertos, empresarios y al público interesado beneficiarse de la investigación

Repasando estos aspectos se pone de manifiesto que muchos de ellos son extrapolables al ámbito de los blogs, además aportando un valor añadido, y es que los ofrece a priori, durante el desarrollo de la investigación.

A pesar de todas estas virtudes, datos como el descenso en la actualización o agregación de nuevos contenidos a los blogs en 2012 (Fundación telefónica, 2013) o que más del 80\% de los mismos poseen una actualización muy escasa o nula (eEspaña, 2012) ponen de manifiesto que algo falla. Esto puede tener que ver con la dificultad de acreditar la calidad de los contenidos de un blog o con la falta de visibilidad, promoción y uso que, en muchos casos hace que los autores pierdan la motivación por mantener sus contenidos actualizados o incluso en algunos casos desistan.

La libertad por parte del autor a la hora de ofrecer y organizar sus contenidos en los blogs que, en principio, puede considerarse una de las principales virtudes y factor desencadenante de su éxito, se convierte también en uno de sus principales problemas. Los blogs, sus contenidos y sus comunidades son difícilmente identificables, clasificables y cuantificables, constituyendo un todo difuso bajo la denominación de blogosfera. Por ejemplo, uno de los portales más importantes sobre blogs de la actualidad, Technorati ${ }^{3}$, incluye todos los blogs "científicos" bajo un único epígrafe denominado -Science- con más de 16.000 blogs en esa categoría, hecho que dificulta la localización de contenidos específicos y su correspondiente puesta en valor.

En al ámbito de la Biblioteconomía y Documentación española (ByD en adelante) la cuestión de los blogs ha sido abordada por diversos autores, tanto a nivel instrumental como a nivel de comunidad. Podemos encontrar algunas aproximaciones como la aportada por Flores y Legerén (2005) que defendieron la existencia de una red social compuesta por bitácoras de Biblioteconomía y Documentación y ciencias afines en español; la aproximación de Ros (2005) que actualizó la anterior y posteriormente siguió planteando la cuestión de la existencia de una comunidad de blogs en nuestro ámbito (Ros, 2008) o incluso llegó a hablar del ocaso de la Biblioblogosfera (Ros, 2008). Becerril (2006) aportó una recopilación de blogs ByD que además incluía una descripción de los mismos; o Torres, Cabezas y Delgado (2008) que publicaron un primer análisis métrico de blogs españoles sobre ByD en el que se establecieron indicadores como número de posts por día o número de comentarios recibidos.

Si bien todas estas aproximaciones aportan un punto de partida y aspectos muy importantes a tener en cuenta, años después se sigue teniendo una imagen difusa de lo que se ha dado en llamar Biblogsfera y poco se ha contrastado sobre su autoría, su estructura, sus relaciones y sus contenidos.

En cualquier caso, como hemos indicado al comienzo de esta introducción, en contra de la opinión de muchos que constantemente vaticinan su final, los blogs siguen siendo una herramienta vigente que ha demostrado un alto nivel de adaptación y flexibilidad. Esto, unido al resto de sus características, forma un conjunto difícilmente sustituible como instrumento en el ámbito científico, en especial en una comunidad pequeña como la nuestra, en la que la visibilidad y la cohesión pueden mejorar el impacto de los trabajos y por lo tanto nos hace fuertes ante un contexto de crisis como el actual.

De esta forma, factores como la identificación de autoría, de contenido y estructura, resultan necesarios y determinantes para delimitar su concepto, y a su vez, son una importante ayuda a la hora de aportar visibilidad y uso, todo ello esencial para la puesta en valor de los contenidos blog, de los blogs como herramienta y, en definitiva, de la Biblogsfera como comunidad. 


\section{OBJETIVOS Y METODOLOGÍA.}

En base al estudio "Aproximación a la Biblogsfera española" (Vera, 2013), mediante este artículo se pretende identificar la presencia de los blogs en el ámbito de la Biblioteconomía y Documentación en España, analizando sus elementos, su autoría y sus relaciones con la finalidad de recopilar indicadores que puedan arrojar luz sobre si realmente se puede hablar de una Biblogsfera, en qué consiste y quién la forma.

Como objetivos específicos marcamos:

- Identificar blogs españoles relacionados con el ámbito de la ByD

- Identificar y analizar sus relaciones

- Recopilar criterios de valoración de los blogs

- Identificar su contenido, su autoría y el perfil profesional de estos

- Identificar su localización geográfica

Metodológicamente, el primer problema que afrontamos es desde el punto de vista de la muestra. Si bien se han podido encontrar distintas relaciones de blogs vinculadas al ámbito de la Biblioteconomía y Documentación, en ocasiones se fundamentan en directorios sesgados y en otras no se hace referencia al criterio de selección o éstos se basan en la experiencia personal y el uso. Así, finalmente se propone una encuesta entre profesionales de la ByD para identificar de manera objetiva la muestra inicial y conseguir una aproximación lo más rigurosa posible.

Utilizando la plataforma de encuestas de la Universidad de Murcia se envía una encuesta estructurada a más de 500 profesionales vinculados a la ByD durante el periodo 01/05/2013 - 31/05/2013. En ella se pide que identifiquen y valoren el uso que hacen de los blogs desde esta perspectiva profesional. La encuesta nos ayuda también a repasar el estado de la cuestión, averiguando cómo valoran estos profesionales el uso de los blogs. Se consigue recopilar un total de 94 encuestas.

Una vez recopilada la muestra, planteamos qué metodología utilizar para su análisis. Si bien la mayor parte de las metodologías encontradas suelen tener un carácter métrico, los aspectos que nos interesa evaluar son de carácter cualitativo y para ello planteamos identificar dentro de la estructura del blog los elementos que puedan aportar algún tipo de información relacionada con la autoría, los contenidos y las relaciones existentes entre blogs.

Además, con la finalidad de poder aportar criterios de clasificación para los diferentes datos que posteriormente se ofrecerán también se recopila información de carácter cuantitativo.

Así, identificamos los siguientes datos de interés para nuestro estudio:

De carácter cualitativo

- Fecha primer post: tomamos como inicio de la actividad del blog la fecha en la que aparece publicado el primer post en la plataforma que utilice actualmente. No se tiene en cuenta, por lo tanto, si el blog ha cambiado de plataforma y no ha exportado los antiguos posts.

- Número de posts: esta información es meramente descriptiva e indica el número de post que se han publicado en cada blog desde la publicación del primero hasta el día de la consulta de sus datos (de marzo a mayo de 2013).

- Posts mensuales: el cálculo de la productividad se ha realizado teniendo en cuenta el número total de posts del blog y el número de meses transcurrido entre el primer y último post ( $\sum$ Posts / [( (Fecha ultimo post - Fecha primer post)/365) x 12]). El dato obtenido es el número medio de posts publicados mensualmente.

De carácter cuantitativo

- Autoría: dato esencial para la identificación de un blog, suele aparecer explícitamente en forma de página "acerca de" o "sobre el autor", aunque en algunos casos se recurre al post de presentación del blog o incluso a referencias externas.

- Perfil profesional: al tratarse de la identificación de una comunidad de carácter profesional es de especial importancia intentar identificar el perfil profesional de esta autoría, para ello se ha recurrido a la propia presentación y a los directorios profesionales Linked $\ln ^{4}$ y al directorio especializado Exit ${ }^{5}$.

- Localización: por otra parte, con la finalidad de poder identificar los principales núcleos de producción en España y, ante la dificultad de conocer la localización geográfica de cada autor, se intenta identificar a través de su puesto de trabajo. 
- Etiquetas y categorías: elemento que forma parte de la estructura nativa de los blogs y que consiste en un sistema de clasificación por el que el autor asigna descriptores que identifican de alguna manera el contenido de cada post. Por lo tanto, nos aporta una valiosa información de las materias y temáticas tratadas.

- Blogroll: consiste en una ventana (widget) en la que el autor indica una lista de enlaces a otros blogs con diferentes criterios. Éstos pueden ser por afinidad, por interés común, por amistad... Este elemento, comúnmente extendido en las distintas plataformas de blogs, tiene una especial importancia para poder evaluar las relaciones entre los distintos blogs. Por otra parte, los blogrolls nos van a permitir también completar la muestra mediante la identificación de nuevos blogs.

Estos datos se recopilan en una base de datos para su posterior análisis y evaluación y a partir de ellos elaborar los resultados presentados a continuación.

\section{RESULTADOS.}

Como principales resultados de la encuesta presentamos los siguientes datos:

En relación al uso que los profesionales de la ByD hacen de los blogs, un 35,11\% consultan blogs ByD, un 14,89\% publican o han publicado contenidos en algún blog ByD y un 36,17\% han mostrado interés por ser informados del resultado de este trabajo.

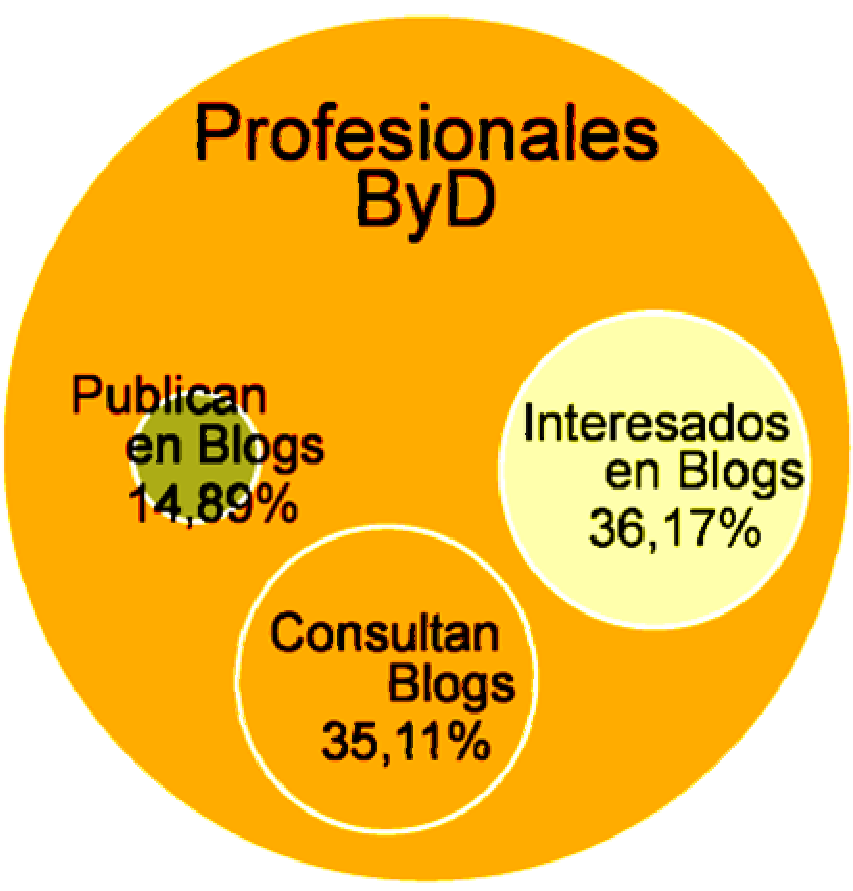

Gráfico 1. Uso de blogs por profesionales ByD.

En relación a la identificación de los blogs, conviene aclarar que la encuesta se realiza en un sentido amplio, ya que se pretende obtener el mayor número de resultados posible. En algunos casos los blogs identificados no se ajustan a los requisitos más exhaustivos de nuestro estudio. Por tanto, se descartan algunos con los siguientes criterios: blogs cerrados o inactivos, blogs que están fuera del ámbito geográfico (España), blogs vinculados a publicitar y/o promocionar servicios y blogs de otros ámbitos como la informática o la comunicación, llegando a una muestra final de 123 blogs.

Se presenta la relación de blogs que han sido mencionados en dos o más encuestas y se indica si alguien los señala como imprescindibles. 


\begin{tabular}{|c|c|c|c|c|}
\hline Pos. & Blog & Url & Menciones & $\begin{array}{l}\text { Marcado como } \\
\text { Imprescindible }\end{array}$ \\
\hline 1 & Biblogtecarios & http://www.biblogtecarios.es/ & 10 & Sí \\
\hline 2 & Biblioblog, 4a edición & http://biblioblog.org/ & 5 & Sí \\
\hline 3 & Deakialli docu-Mental & http://www.deakialli.com/ & 5 & Sí \\
\hline 4 & $\begin{array}{l}\text { Bibliotecarios 2020 (antes } \\
\text { Bibliotecarios 2.0) }\end{array}$ & http://www.nievesglez.com/ & 4 & Sí \\
\hline 5 & RecBib: Recursos Bibliotecarios & http://www.recbib.es/ & 4 & Sí \\
\hline 6 & $\begin{array}{l}\text { Bdig (biblioteques digitals i } \\
\text { cooperació) }\end{array}$ & http://bdig.blogspot.com/ & 3 & Sí \\
\hline 7 & $\begin{array}{l}\text { BRMU - Biblioteca Regional de } \\
\text { Murcia }\end{array}$ & http://brmu.blogspot.com/ & 3 & \\
\hline 8 & Documanía 2.0 & http://www.documania.info/ & 3 & \\
\hline 9 & Documentación & http://www.madrimasd.org/blogs/documentacion/ & 3 & Sí \\
\hline 10 & Julián Marquina & http://www.julianmarquina.es/ & 3 & \\
\hline 11 & SEDIC blog & http://blog.sedic.es/ & 3 & \\
\hline 12 & SocialBiblio & http://www.socialbiblio.com/ & 3 & \\
\hline 13 & Bibliotecas 2029 & http://bibliotecas2029.wordpress.com/ & 2 & Sí \\
\hline 14 & Blok de BiD & http://www.ub.edu/blokdebid/ & 2 & Sí \\
\hline 15 & $\begin{array}{l}\text { BNE - Biblioteca Nacional de } \\
\text { España }\end{array}$ & http://blog.bne.es/ & 2 & \\
\hline 16 & Dokumentalistas & http://www.dokumentalistas.com/ & 2 & Sí \\
\hline 17 & EC3noticias & http://ec3noticias.blogspot.com/ & 2 & \\
\hline 18 & EPI Blog & http://www.elprofesionaldelainformacion.com/notas/ & 2 & \\
\hline 19 & $\begin{array}{l}\text { Expediente, web, blog y otros } \\
\text { documentos del montón }\end{array}$ & http://documentalqueridowatson.es & 2 & \\
\hline 20 & $\begin{array}{l}\text { Primer Cuartil (Documentación, } \\
\text { biblioteconomía e información) }\end{array}$ & http://alvarocabezas.com/ & 2 & \\
\hline 21 & Universo Abierto & http://www.universoabierto.com/ & 2 & Sí \\
\hline
\end{tabular}

Tabla I. Blogs más referenciados por profesionales ByD.

Como principales resultados del análisis de los distintos elementos indicados en la metodología sobre cada blog se presentan en la tabla II, que incluye el ranking de los blogs más mencionados en los blogrolls., así como el número de menciones. 


\begin{tabular}{|c|c|c|c|}
\hline Pos. & Blog & Url & Blogroll \\
\hline 1 & Deakialli docu-Mental & http://www.deakialli.com/ & 18 \\
\hline 2 & Biblogtecarios & http://www.biblogtecarios.es/ & 16 \\
\hline 3 & Biblioblog, 4a edición & http://biblioblog.org/ & 12 \\
\hline 4 & RecBib: Recursos Bibliotecarios & http://www.recbib.es/ & 12 \\
\hline 5 & SEDIC blog & http://blog.sedic.es/ & 12 \\
\hline 6 & $\begin{array}{l}\text { Bibliotecarios } 2020 \text { (antes Bibliotecarios } \\
2.0 \text { ) }\end{array}$ & http://www.nievesglez.com/ & 11 \\
\hline 7 & Javier Leiva (Catorze) & http://www.javierleiva.info/ & 11 \\
\hline 8 & El documentalista enredado & http://www.documentalistaenredado.net/ & 10 \\
\hline 9 & SocialBiblio & http://www.socialbiblio.com/ & 9 \\
\hline 10 & Julian Marquina & http://www.julianmarquina.es/ & 8 \\
\hline 11 & Documanía 2.0 & http://www.documania.info/ & 7 \\
\hline 12 & Archinfo 2.0 & http://archinfo20.es/ & 6 \\
\hline 13 & Blog EPI & http://www.elprofesionaldelainformacion.com/notas/ & 6 \\
\hline 14 & Docu ¿qué? & http://entreolasdeinformacion.blogspot.com/ & 6 \\
\hline 15 & Garabuya (APEI) & http://garabuya.blogspot.com/ & 6 \\
\hline 16 & Libros y bitios (el futuro del libro) & http://jamillan.com/librosybitios/ & 6 \\
\hline 17 & ThinkEPI & http://www.thinkepi.net/ & 6 \\
\hline 18 & Bauen blog & http://www.bauenblog.info/ & 5 \\
\hline 19 & Bibliometría (Antiguo BiDo) & http://www.bibliometria.com/ & 5 \\
\hline 20 & $\begin{array}{l}\text { COABDM - Archiveros, Bibliotecarios y } \\
\text { Documentalistas de Madrid }\end{array}$ & http://coabdm.wordpress.com/ & 5 \\
\hline 21 & Dokumentalistas & http://www.dokumentalistas.com/ & 5 \\
\hline 22 & Gestión Documental para Gente Normal & http://gestiondocumentalparagentenormal.com/ & 5 \\
\hline
\end{tabular}

Tabla II. Relación de blogs más mencionados en los blogrolls.

En relación a la autoría, de los 123 blogs objeto de estudio se han identificado un total de 283. De ellas 32 se identifican como entidades y 251 como personas. A continuación se presenta la relación de autores indicando el número de blogs en los que escriben. 


\begin{tabular}{|c|c|}
\hline Autor & $\begin{array}{l}N^{0} \text { de Blogs en } \\
\text { los que escribe }\end{array}$ \\
\hline Arroyo Vázquez, Natalia & 4 \\
\hline Guallar, Javier & 4 \\
\hline Leiva Aguilera, Javier & 4 \\
\hline Marquina Arenas, Julian & 4 \\
\hline Torres Salinas, Daniel & 4 \\
\hline Anglada i de Ferrer, Lluís M. & 3 \\
\hline Baiget, Tomàs & 3 \\
\hline Codina Bonilla, José Lluís & 3 \\
\hline Giménez Toledo, Elea & 3 \\
\hline Merlo Vega, José Antonio & 3 \\
\hline Millán González, José Antonio & 3 \\
\hline Tejada Artigas, Carlos Miguel & 3 \\
\hline Tosete Herranz, Francisco & 3 \\
\hline Tramullas, Jesús & 3 \\
\hline Abadal Falgueras, Ernest & 2 \\
\hline Bañal Teijido, María Luz & 2 \\
\hline Baños Moreno, María José & 2 \\
\hline Benítez Acosta, María & 2 \\
\hline Cabezas Clavijo, Álvaro & 2 \\
\hline Calderón Rehecho, Andoni & 2 \\
\hline Castro, Pablo de & 2 \\
\hline Cordón García, José Antonio & 2 \\
\hline Delgado López-Cózar, Emilio & 2 \\
\hline Eíto Brun, Ricardo & 2 \\
\hline García-Puente Sánchez, María & 2 \\
\hline Garea García, Natalia & 2 \\
\hline Gionés Valls, Aina & 2 \\
\hline González, Nieves & 2 \\
\hline Hassan Montero, Yusef & 2 \\
\hline Juárez Urquijo, Fernando & 2 \\
\hline López de Quintana Saenz, Eugenio & 2 \\
\hline Lozano, Roser & 2 \\
\hline Muñoz Romano, Jose Luis & 2 \\
\hline Olmo García, María Jesús del & 2 \\
\hline Penadés de la Cruz, Honorio & 2 \\
\hline Peset Mancebo, Fernanda & 2 \\
\hline Pons i Serra, Amadeu & 2 \\
\hline Prieto Gutiérrez, Juan José & 2 \\
\hline Robinson García, Nicolás & 2 \\
\hline Rodríguez Yunta, Luis & 2 \\
\hline Sánchez García, María Isabel & 2 \\
\hline Saorín Pérez, Tomás & 2 \\
\hline Serrano Cobos, Jorge & 2 \\
\hline Serrano Muñoz, Jordi & 2 \\
\hline Urbano Salido, Cristóbal & 2 \\
\hline Valseca Gómez, María Jesús & 2 \\
\hline Villapalos Pardiñas, Victor & 2 \\
\hline
\end{tabular}

Tabla III. Autores con número de blogs activos en los que escriben.

En relación al perfil profesional de los autores, se han podido describir 237 perfiles (lo que supone un 94,42\%). Además se consigue identificar la filiación institucional y el puesto que desempeñan de 199 (79,28\% de puestos de trabajo localizados). 
A continuación se muestra una gráfica con las entidades a las que pertenecen los autores (con una incidencia de al menos dos autores).

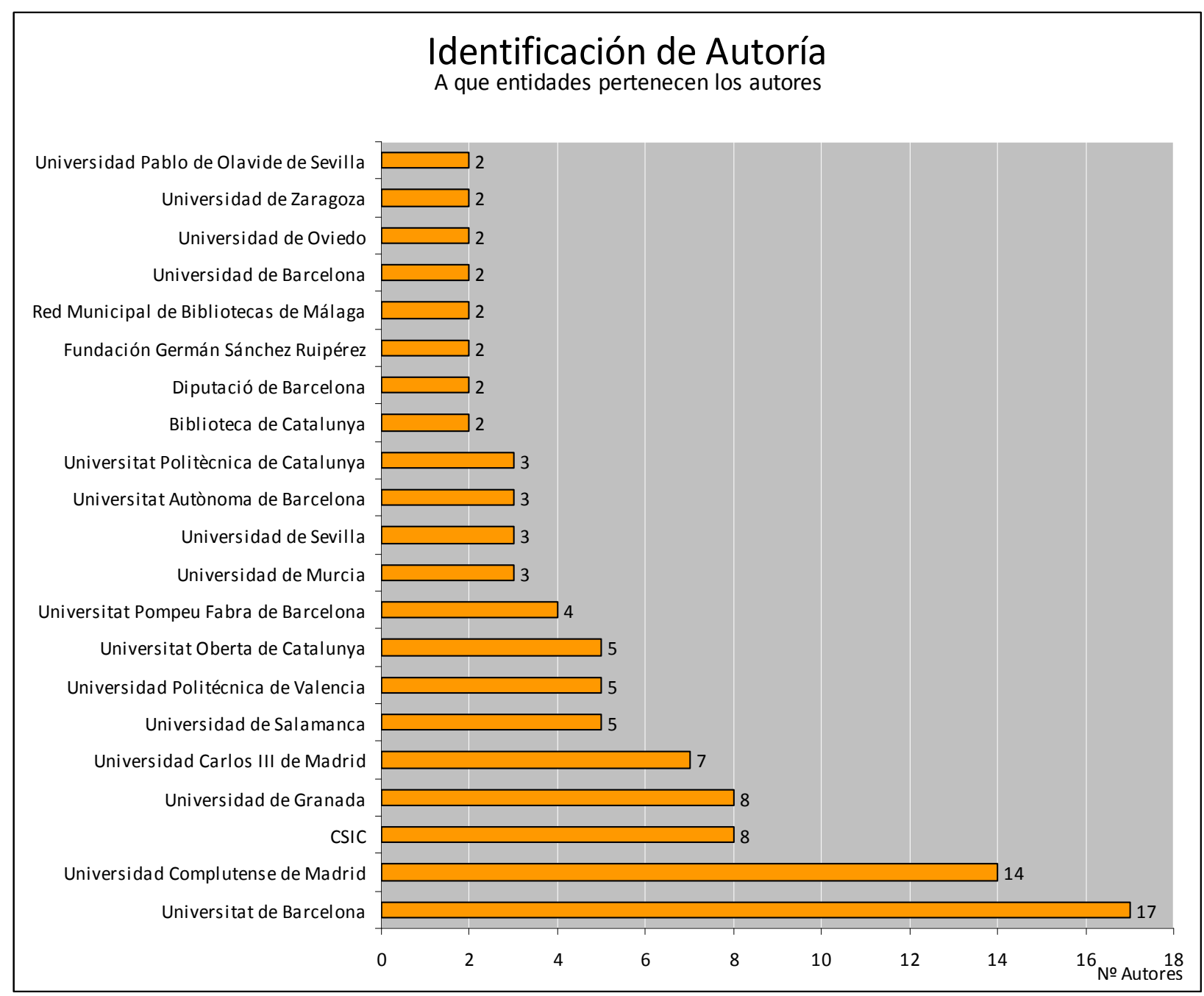

Gráfico 2. Entidades en las que trabajan los autores de blogs de la Biblogsfera.

Respecto a la localización geográfica, se ha asignado a cada autor la localización de su puesto de trabajo y a cada blog la localización de su autor.

Con los datos recopilados, es un hecho que la Biblogsfera se centra principalmente en Barcelona y Madrid, lo que quizá tenga que ver con la realidad de que son dos áreas donde se aglutina gran número de profesionales de nuestro campo, además de concentrar varias universidades lo que, como hemos podido comprobar en el punto anterior respecto al perfil profesional de la autoría, es un factor determinante y tiene una fuerte vinculación. De esta manera configuramos el siguiente mapa del gráfico 3. 


\section{Principales núcleos de la Biblogsfera española}

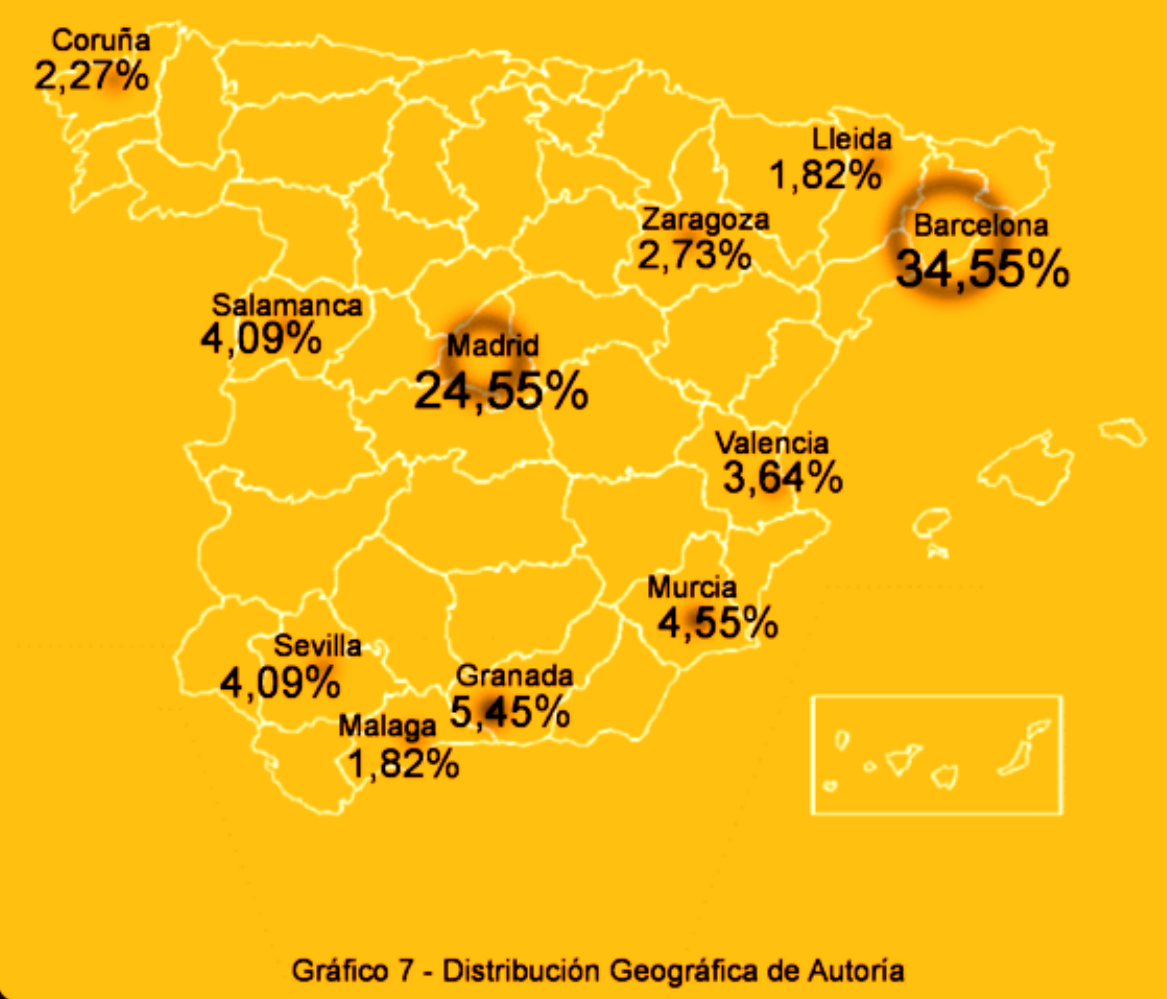

Gráfico 3. Distribución geográfica de autoría.

Respecto al contenido, a pesar de que la información de etiquetas y categorías no esta disponible en todos los blogs, se ha recopilado un total de 638 categorías y de 4.354 etiquetas indicando en lo posible el número de posts incluidos bajo cada una de ellas. Éstas se han agrupado y escalado según la perspectiva de "Unidades de Información del "Tesauro de Biblioteconomía y Documentación" del (CSIC). De esta forma llegamos al siguiente listado en el que se incluye el porcentaje que representa cada etiqueta sobre el total.

- Archivos $(8,16 \%)$

- Bibliotecas $(22,52 \%)$

- Centros de documentación $(5,46 \%)$

- Servicios de información (2,26\%)

- Otras Unidades de Información (2,01\%)

- Materias transversales $(18,82 \%)$

- Catalogación $(0,5 \%)$

- Digitalización y preservación digital $(2,63 \%)$

- TICs y Sociedad de la Información $(12,42 \%)$

- Redes Sociales $(5,65 \%)$

- Profesión (19,57\%)

\section{CONCLUSIONES.}

Analizados los resultados de las encuestas a profesionales de la $\mathrm{ByD}$, es decir, las referencias de los profesionales en intersección con las referencias de los blogsroll, es decir, las referencias de los autores, encontramos que entre los 10 primeros resultados de cada tabla hay una coincidencia de 7 blogs $(70 \%)$. 


\begin{tabular}{|c|l|c|}
\hline Pos. & $\begin{array}{c}\text { Blogs más referenciados en los } \\
\text { blogsroll }\end{array}$ & Menciones \\
\hline 1 & Deakialli docu-Mental & 18 \\
\hline 2 & Biblogtecarios & 16 \\
\hline 3 & SEDIC blog & 12 \\
\hline 4 & Biblioblog, 4a edición & 12 \\
\hline 5 & RecBib: Recursos Bibliotecarios & 12 \\
\hline 6 & Javier Leiva (Catorze) & 11 \\
\hline 7 & $\begin{array}{l}\text { Bibliotecarios 2020 (antes } \\
\text { Bibliotecarios 2.0) }\end{array}$ & 11 \\
\hline 8 & El documentalista enredado & 10 \\
\hline 9 & SocialBiblio & 9 \\
\hline 10 & Julian Marquina & 8 \\
\hline
\end{tabular}

\begin{tabular}{|c|l|c|}
\hline Pos. & $\begin{array}{c}\text { Blogs más referenciados en la } \\
\text { encuesta }\end{array}$ & Menciones \\
\hline 1 & Biblogtecarios & 10 \\
\hline 2 & Biblioblog, 4a edición & 5 \\
\hline 3 & Deakialli docu-Mental & 5 \\
\hline 4 & $\begin{array}{l}\text { Bibliotecarios 2020 (antes } \\
\text { Bibliotecarios 2.0) }\end{array}$ & 4 \\
\hline 5 & RecBib: Recursos Bibliotecarios & 3 \\
\hline 6 & $\begin{array}{l}\text { Bdig (biblioteques digitals i } \\
\text { cooperació) }\end{array}$ & 3 \\
\hline 7 & SocialBiblio & 3 \\
\hline 8 & SEDIC blog & 3 \\
\hline 9 & Documentación & 3 \\
\hline 10 & Documanía 2.0 & \\
\hline
\end{tabular}

Tabla IV Comparativa blogs más referenciados blogsroll / encuesta.

Esta coincidencia se repite a lo largo de todo el listado de referencias, por lo que estamos en disposición de afirmar que, a pesar de su falta de concreción, tanto desde la perspectiva de los profesionales de la ByD, como de la perspectiva de los autores, hay una imagen tácita coincidente, que agrupa de manera natural los mismos blogs.

Respecto a la autoría, su identificación ha resultado determinante para establecer el carácter profesional de esta comunidad y como indicador de la calidad de los contenidos ofrecidos en la misma. Del análisis de los datos recopilados se desprende que los autores son en su práctica totalidad profesionales de la Biblioteconomía y la Documentación, principalmente personal docente e investigador universitario y del CSIC (40,32\%), profesionales de bibliotecas y archivos de centros públicos, universitarios y privados $(25,69 \%)$ y otros profesionales de centros de documentación principalmente del ámbito privado (12,25\%), en un alto grado de reconocido prestigio, teniendo este dato una importancia adicional ya que, como veíamos en la introducción, este factor le da carácter científico a la comunidad.

Respecto a su contenido y las relaciones indicadas en los blogrolls, como hemos señalado en los resultados, hay una agrupación natural por temáticas coincidentes o afines e inherente a la muestra pertenecientes al ámbito de la Biblioteconomía y la Documentación. Y, si bien sus relaciones reflejan el comportamiento de su patrón de comunicación, es decir, multidireccional y descentralizado, es interesante comprobar que los blogs se señalan entre si principalmente por esta afinidad en cuanto a contenidos, pudiéndose identificar incluso otros corpúsculos mas pequeños, principalmente en materia de bibliotecas y en materia de archivos. No se puede establecer esta relación teniendo en cuenta la procedencia geográfica.

Por lo tanto, y teniendo en cuenta todas las consideraciones anteriores, estamos en disposición de afirmar que realmente existe una red de blogs especializada, agrupada de forma natural por el interés común de autores y usuarios en alguna de las áreas de la Biblioteconomía y la Documentación, con una estructura descentralizada, multidireccional e interactiva y cuyo contenido es producido y dirigido a profesionales de este ámbito ${ }^{6}$.

\section{NOTAS}

\footnotetext{
${ }^{1}<$ http://en.wordpress.com/stats/>

${ }^{2}$ Corriente que persigue el Acceso Abierto a la información científica con el único requisito de su revisión por pares.

$3<$ http://technorati.com/blogs/directory/>.

${ }^{4}$ LinkedIn. Directorio de profesionales. Disponible en: $<$ http://es.linkedin.com $>$.

${ }^{5}$ Exit. Directorio de expertos en el tratamiento de la información. Disponible en: <www.directorioexit.info >.

${ }^{6}$ Como línea posterior al TFG "Aproximación a la BIBLOGSFERA española: composición, autoría, estructura, contenidos y definición", dirigido por Francisco Javier Martínez Méndez, disponible en: <http://digitum.um.es/xmlui/handle/10201/36967>, donde se puede ampliar información sobre este tema, se ha creado un directorio de blogs ByD disponible en <www.biblogsfera.com $>$ que pretende visibilizar, cohesionar y dinamizar esta comunidad.
} 


\section{BIBLIOGRAFÍA.}

BECERRIL GONZÁLEZ, V.; et al. Blogs de Biblioteconomía y Documentación en España. Revista Española de Documentación Cientifica, 2006, vol. 29, no 4 [en línea]. Disponible en: <http://eprints.rclis.org/8903/> [Consultado: 17 diciembre 2013].

CEREZO, J.M. La blogosfera hispana: pioneros de la cultura digital. Madrid: Fundación FranceTelecom, 2006 [en línea]. Disponible en: <http://fundacionorange.es/areas/25_publicaciones/la_blogosfera_hispana.pdf $>$ [Consultado: 20 diciembre 2013].

eEspaña informe anual 2012 sobre el desarrollo de de la sociedad de la información en España. Madrid: Fundación Orange, 2012 [en línea]. Disponible en: <http://www.proyectosfundacionorange.es/docs/eE2012.pdf $>$ [Consultado: 4 diciembre 2013].

Estudio sobre uso, interés, conocimiento y percepción de la blogosfera española. ZedDigital, 2007 [en línea]. Disponible en: <http://www.merodeando.com/archivos/Blogosfera_abr07.pdf $>$ [Consultado: 27 diciembre 2013].

FERRADA CUBILLOS, M. Biblogsfera, comunidad de bitácoras cuya temática versa total o parcialmente sobre Biblioteconomía y Documentación. Biblios, 2006, no 24 [en línea]. Disponible en: <http://eprints.rclis.org/8096/>.

FERRADA CUBILLOS, M. Weblogs o bitácoras: un recurso de colaboración en línea para los profesionales de la información. Serie Bibliotecología y Gestión de Información, 2005, no 6 [en línea]. Disponible en: $<$ http://eprints.rclis.org/6787/> [Consultado: 27 diciembre 2013].

FLORES CALVO, B. y LEGERÉN ÁLVAREZ, E. El fenómeno weblog como nuevo medio de comunicación: su incidencia en el campo de la Biblioteconomía y la Documentación. En: La dimensió humana de l'organització del coneixement. 1er Encuentro de Estudiantes de Documentación para la Organización del Conocimiento. $7^{\circ}$ Congreso ISKO-España, Barcelona (España), 6-8 julio 2005. [Conference paper] Disponible en: $<$ http://eprints.rclis.org/20360/> [Consultado: 8 enero 2014].

FRANGANILLO, J. y CATALÁN, M.A. Bitácoras y sindicación de contenidos: dos herramientas para difundir información. BiD: textos universitaris de biblioteconomia i documentació, 2005, $\mathrm{n}^{\mathrm{o}} 15$ [en línea]. Disponible en: $<$ http://www2.ub.edu/bid/consulta_articulos.php?fichero=15frang2.htm > [Consultado: 18 diciembre 2013].

Fundación Telefónica. La Sociedad dé la Información en España 2012. Madrid: Ariel, 2013 [en línea]. Disponible en: $<$ http:/e-libros.fundacion.telefonica.com/sie12/aplicacion_sie/ParteA/pdf/SIE_2012.pdf $>$ [Consultado: 9 diciembre 2013].

La Sociedad en Red 2011. Madrid: Observatorio Nacional de las Telecomunicaciones y la Sociedad de la Información, 2012 [en línea]. Disponible en: $<$ http://www.ontsi.red.es/ontsi/sites/default/files/la_sociedad_en_red_2011_ed2012.pdf $>$ [Consultado: 27 diciembre 2013].

LEIVA AGUILERA, J. Blogs: una herramienta de difusión para profesionales de la información. Revista TK, 2006, $\mathrm{n}^{\circ}$ 18 [en línea]. Disponible en: <http://eprints.rclis.org/12662/> [Consultado: 5 enero 2014].

LEIVA AGUILERA, J. Breve aproximación a los blogs para unidades de información. Thinkepi, 2006 [en línea]. Disponible en: <http://www.thinkepi.net/breve-aproximacion-a-los-blogs-para-unidades-de-informacion> [Consultado: 5 enero 2014].

LLEDÓ, M. El profesional de la información ante los weblogs. En: Contenidos y Aspectos Legales en la Sociedad de la Información (CALSI), Valencia, 23 $\square 24$ octubre 2003 [en línea]. Disponible en: <http://eprints.rclis.org/4291/> [Consultado: 9 diciembre 2013].

MERLO VEGA, J.A. y SORLI ROJO, A. Weblogs: un recurso para los profesionales de la información. Revista Española de Documentación Cientifica, 2003, vol. 26, n ${ }^{\mathrm{o}} 2$ [en línea]. Disponible en: $<$ http://eprints.rclis.org/6787/1/serie_6.pdf> [Consultado: 10 diciembre 2013].

ROS MARTÍN, M. Análisis de la biblioblogosfera hispana. El documentalista enredado, 2005 [Blog Internet]. Disponible en: <http://www.documentalistaenredado.net/biblioblogosfera/> [Consultado: 18 diciembre 2013].

ROS MARTÍN, M. Un lustro de Biblioblogosfera. El documentalista enredado, 2006 [Blog Internet]. Disponible en: $<$ http://www.documentalistaenredado.net/441/un-lustro-de-biblioblogosfera/> [Consultado: 22 diciembre 2013].

ROS MARTÍN, M.¿El ocaso de la Biblioblogosfera? El documentalista enredado, 2008 [Blog Internet]. Disponible en: <http://www.documentalistaenredado.net/682/el-ocaso-de-la-biblioblogosfera/> [Consultado: 19 noviembre 2013].

ROS MARTÍN, M. Biblioblogosfera $\square$ Comunidad virtual distribuida de bibliotecarios y documentalistas. En: $6^{\circ}$ Curso de Edición $\square$ Sociología de la Literatura, Valencia, 19/06/2008. [Presentación]. Disponible en: $<$ http://eprints.rclis.org/11765/> [Consultado: 10 enero 2014].

State of the Blogosphere 2011. Technorati, 2012 [en línea]. Disponible en: <http://technorati.com/socialmedia/feature/state-of-the-blogosphere-2011> [Consultado: 18 diciembre 2013]. 
SWAN, A. Directrices para Políticas de desarrollo y promoción del acceso abierto. UNESCO, 2013.

SWAN, A. Open Access and the progress of science (in Spanish, en Espanol). American Scientist, 2007, n ${ }^{\circ}$ 95, p. 197199. <http://eprints.soton.ac.uk/263860/> [Consultado: 18 noviembre 2013].

TORRES SALINAS, D.; CABEZAS CLAVIJO, A. y DELGADO LÓPEZ $\square$ COZAR, E. Análisis métrico de los blogs españoles de Biblioteconomía y Documentación. El Profesional de la Información, 2008. Disponible en: $<$ http://www.elprofesionaldelainformacion.com/contenidos/2008/enero/04.html> [Consultado: 18 noviembre 2013].

TORRES SALINAS, D. y CABEZAS CLAVIJO, A. Los blogs como nuevo medio de comunicación científica. En: III Encuentro Ibérico de Docentes e Investigadores en Información y Documentación, Salamanca, $5 \square 7$ mayo 2008. [Conferencia]. Disponible en: <http://ec3.ugr.es/publicaciones/Torres-Salinas,_Daniel_y_CabezasClavijo,_Alvaro._Los_blogs_como_nuevo_medio_de_comunicacion_cientifica.pdf $>$ [Consultado: 18 noviembre 2013].

VERA BACETA, M.A. Aproximación a la Biblogsfera española: composición, autoría, estructura, contenidos y definición. Trabajo Fin de Grado, Universidad de Murcia (España), 2013. Disponible en: $<$ http://eprints.rclis.org/20757/> [Consultado: 18 noviembre 2013].

ZIVKOVIC, B. Publishing hypotheses and data on a blog - is it going to happen on science blogs? Science \& Politics, 2006 [Blog internet]. Disponible en $<\mathrm{http} / /$ sciencepolitics.blogspot.com/2006/04/publishing-hypotheses-and-dataon-blog.html> Consultado: 19 noviembre 2013]. 